\title{
Treatment with either leflunomide or adalimumab reduces anaemia in patients with rheumatoid arthritis
}

\author{
IONE C.P. PEREIRA ${ }^{1}$, NÁGILA C.F. SOUSA ${ }^{1}$, DOMINGOS M.S. PEREIRA ${ }^{1}$, SAULO J.F. MENDES ${ }^{1}$, \\ THAYANNE F. MUNIZ ${ }^{1}$, VALDERLANE L.P. COLARES ${ }^{1}$, BRUNA L.R. SILVA ${ }^{1}$, CINARA R.A.V. MONTEIRO ${ }^{1,2}$, \\ MAHIBA M.R.S. MARTINS ${ }^{3}$, ANITA M.R. FERNANDES ${ }^{4}$ and ELIZABETH S. FERNANDES ${ }^{1}$ \\ ${ }^{1}$ Programa de Pós-Graduação, Universidade Ceuma, Rua Josué Montello, 1, \\ Bairro Renascença II, 65075-120 São Luis, MA, Brazil \\ ${ }^{2}$ Universidade Federal do Maranhão, Av. dos Portugueses, 1966, Vila Bacanga, 65085-580 São Luís, MA, Brazil \\ ${ }^{3}$ Centro de Especialidades Médicas Vinhais, Av. 02, Vinhais, 65070-000 São Luís, MA, Brazil \\ ${ }^{4}$ Curso de Graduação em Ciência da Computação, Universidade do Vale do Itajaí, Campus \\ Kobrasol, Km 207, MundoCar Shopping, Kobrasol, 88102-700 São José, SC, Brazil
}

Manuscript received on February 6, 2017; accepted for publication on August 2, 2017

\begin{abstract}
Rheumatoid arthritis is a chronic disease of the joints, which causes joint pain and disability. Anaemia is a frequent extra-articular manifestation in rheumatoid arthritis, affecting $30-70 \%$ of the patients; presenting a negative impact on patient's quality of life. Some of the drugs used in rheumatoid arthritis treatment improve anaemia; but little is known on the beneficial effects of the anti-rheumatic leflunomide or the anti$\mathrm{TNF} \alpha$ adalimumab, in this parameter. We investigated the incidence of anaemia in rheumatoid arthritis patients treated or not with leflunomide or adalimumab. We also assessed whether anaemia correlates with disease activity. Anaemia was present in patients who had just been diagnosed with rheumatoid arthritis and had never taken disease modifying agents or biologicals (non-specific therapy group), but not in those taking either leflunomide or adalimumab. The erythrocyte sedimentation rate was increased in patients with non-specific therapy in comparison with those taking either leflunomide or adalimumab. Anaemia correlated with increased erythrocyte sedimentation rate. We suggest that leflunomide and adalimumab may be useful in treating anaemia in patients with rheumatoid arthritis.
\end{abstract}

Key words: anaemia, rheumatoid arthritis, leflunomide, adalimumab.

\section{INTRODUCTION}

Rheumatoid arthritis (RA) is a chronic disease of the joints, which causes joint pain and disability. Disease progression is characterized by intra-

Correspondence to: Elizabeth Soares Fernandes

E-mail: elizabeth.soares@ceuma.br

* Contribution to the centenary of the Brazilian Academy of Sciences. articular (pain and loss of joint function) and extra-articular manifestations of the disease. Anaemia is one of the most common extra-articular manifestations of RA, affecting $30-70 \%$ of the patients, and it has been linked to reduced quality of life (Song et al. 2013, Ganna 2014).

Also known as anaemia of inflammation, it can be triggered by increased release of cytokines, 
especially IL-6, in RA patients. Indeed, cytokine generation in RA has been associated with decreased iron availability, and increased absorption and retention within reticulo-endothelial cells; in addition to reduced numbers of red blood cells (for review see: Moreland and Curtis 2009, Masson 2011). Although lowering of haemoglobin levels was found not be associated with chronic fatigue in RA patients, this response has been highly associated with disease activity (Singh et al. 2014).

RA specific treatment comprises diseasemodifying anti-rheumatic drugs (DMARDs) and biologicals that aim to reduce joint inflammation and pain, thus attenuating disease progression. Although therapy targets the intra-articular manifestations of disease, some anti-rheumatic drugs are suggested to be useful in treating the extra-articular disease. These include some anti$\mathrm{TNF} \alpha$ inhibitors such as anakinra, rituximab (Nyhäll-Wåhlin et al. 2012) and etanercept (Calisto Pérez et al. 2012); and DMARDs such as methotrexate when used alone or in combination with a biological (Doyle et al. 2009). Indeed, these drugs were suggested to improve haemoglobin levels, whilst reducing rheumatoid nodules.

The DMARD leflunomide (LFN) and the antiTNF $\alpha$ adalimumab (ADA) are currently used to treat RA in order to reduce joint pain and inflammation, however, little is known on the beneficial effects of these drugs in improving anaemia in RA patients. Here, we investigated the incidence of anaemia in RA patients treated or not with LFN or ADA. We also assessed whether anaemia correlates with different haematological and demographic parameters in these patients.

\section{MATERIALS AND METHODS}

\section{PATIENTS}

A total of 40 patients (men and women) aged $\geq$ 30 years and clinically diagnosed with RA, were recruited for participation in the study. Patients included those who have just been diagnosed with RA but naïve for DMARDs and biologicals $(n=10)$; patients under LFN (20 mg/day, per os, $n=15$ ) and patients under biological (ADA; $40 \mathrm{mg}$ every 2 weeks, subcutaneously, $n=15$ ) therapy. Patients presented a score $\geq 6$ on the 2010 ACREULAR Classification Criteria For Rheumatoid Arthritis as previously detailed. Healthy subjects $(n=15)$ were used as controls and included those who had no history of recent infections, malignancy or other autoimmune diseases and no present or previous use of DMARDs, biologicals or experimental drugs. We recorded demographics (age, gender and duration of disease), clinical (number of affected joints and presence or absence of rheumatoid nodules), and laboratory data. For laboratory data collection, five millilitres $(\mathrm{ml})$ of peripheral blood were collected from each patient, in tubes containing ethylenediamine tetraacetic acid (EDTA) and used for automated analysis of red blood cell numbers, haemoglobin, haematocrit and erythrocyte sedimentation rate (ESR). The study was reviewed and approved by the Human Research Ethics Committee of the Universidade CEUMA and was performed in compliance with the Declaration of Helsinki. A written informed consent was obtained from each participant.

\section{DATA ANALYSIS}

The size of the experimental groups was calculated according to power analysis calculations in which power was $\geq 80 \%$. Data are represented as mean and individual values obtained from each subject. Accordingly, we used parametric (ANOVA followed by Bonferroni's test) test to determine the significance of differences between groups considering their mean \pm SD values. Correlations between the different parameters were determined using Spearman's rho. Statistical analysis was undertaken using IBM SPSS Statistics 20. $p$ values $<0.05$ were considered statistically significant. 


\section{RESULTS}

\section{SUBJECT CHARACTERISTICS}

The majority of the arthritic population was comprised by women $(75 \% ; 30$ out of 40$)$. Arthritic patients had an average age of 50 years, with those under anti-rheumatic therapy presenting the highest averages (mean \pm SD were as follows: 40.5 \pm 8.4 for the NST group; $54.6 \pm 12.1$ and $54.9 \pm$ 10.9 , for the patients treated with LFN or ADA; respectively). Recently diagnosed arthritic patients (those receiving no specific treatment with antirheumatic drugs, NST group) presented disease for less than 2 years (mean \pm SD of $44.9 \pm 8.0$ ) whilst those under treatment with LFN or ADA had disease symptoms for longer than 10 years (mean \pm SD of $54.6 \pm 12.1$ and $51.0 \pm 13.6$; for LFN and ADA patients, respectively). The number of swollen joints was higher in patients recently diagnosed $(11.0 \pm 2.7)$ in comparison with those of patients under LFN $(8.3 \pm 3.1)$ or $\mathrm{ADA}(7.3 \pm 2.9)$ therapy. The incidence of rheumatoid nodules was higher in patients receiving LFN or ADA ( $53 \%$ and $80 \%$, respectively), in comparison with patients recently diagnosed (30\%) with RA. The majority of the subjects composing the population of healthy subjects were women $(73 \%)$ with an average age of 40 years.

\section{TREATMENT WITH EITHER LFN OR ADA} ATTENUATES ANAEMIA IN RA PATIENTS

As shown in Figure 1a, healthy subjects and RA patients presented similar numbers of red blood cells. However, the NST group presented lower levels of haemoglobin (15\%) in comparison with those of healthy subjects (Figure 1b). A similar reduction was observed for hematocrit although this response was not significant (Figure 1c). RA patients treated with either LFN or ADA presented haemoglobin and hematrocit levels similar to those found in healthy subjects (Figure 1b, c). Additionally, the NST group exhibited higher ESR (16.7-fold increase) when compared with healthy subjects (Figure 1d). LFN or ADA administration in RA patients decreased ESR by $64 \%$ and $55 \%$, respectively. Haemoglobin and hematocrit levels strongly and positively correlated with the numbers of red blood cells in healthy subjects $(r=0.946$ for both parameters; $p<0.01$ ), the NST group ( $r=0.729$ and $r=0.848$; respectively, $p<0.05$ ), and patients receiving LFN ( $r=0.839$ and $r=0.31$; respectively, $p<0.05)$ or $\operatorname{ADA}(r=0.896$ and $r=0.815$; respectively, $p<0.05)$. Haemoglobin correlated with hematocrit levels in RA patients, but not healthy subjects. Correlations were $r=0.628(p<0.05), r=0.977$ $(p<0.01)$ and $r=0.937(p<0.01)$, for the NST, LFN and ADA groups; respectively. Positive but weaker correlations were also observed between the numbers of affected joints and the levels of haemoglobin in the NST and LFN groups only ( $r=0.446$ and $r=0.451$, respectively). No significant correlations were found between age, gender or disease time and the evaluated haematological parameters in any of the groups.

\section{DISCUSSION}

Anaemia is common in RA patients and it has been linked with disease activity (Smyrnova 2014). At least three pathophysiological pathways are associated with this extra-articular manifestation of RA, all involving cytokine production. Indeed, increased activation of inflammatory cells during the establishment and progression of disease, leads to an excessive production of cytokines such as TNF $\alpha$, IL-1 $\beta$ and IL-6 (Moreland and Curtis 2009). In turn, these cytokines act on erythropoietic progenitor cells promoting haemolysis and subsequent reduction in the numbers of circulating red blood cells (Papadaki et al. 2002, Masson 2011). It has also been suggested that cytokine production in RA leads to reduction in the availability of iron (Spivak 2000, Masson 2011). This response has been associated with increased 

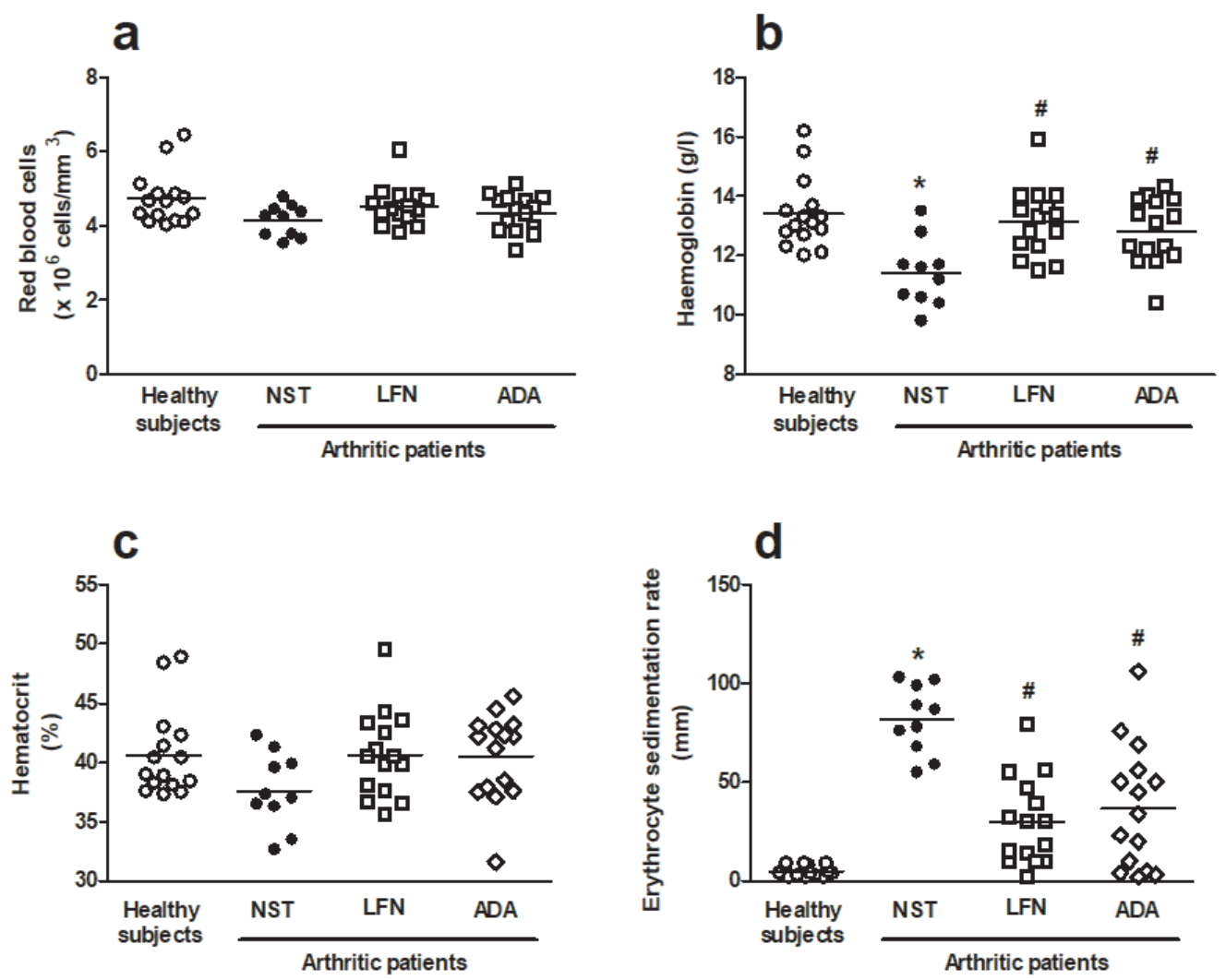

Figure 1 - Peripheral red blood cells (a), haemoglobin (b), hematocrit (c) levels and erythrocyte sedimentation rate (d) in patients with rheumatoid arthritis (RA) treated or not with either leflunomide (LFN; $n=15$ ) or adalimumab (ADA; $n=15$ ), in comparison with patients recently diagnosed with RA but not yet receiving specific treatment with anti-rheumatic drugs (NST group; $n=10)$ and healthy subjects $(n=15)$. Data are expressed as mean and individual values obtained from each subject. Statistical differences between groups considered the mean \pm SD values of each group. $* p<0.05$, differs from healthy subjects; ${ }^{\#} p<0.05$, differs from the NST group.

production of lactoferrin by activated neutrophils and increased iron absorption and retention within cells of the reticulo-endothelial system, leading to low circulating iron levels and low serum binding capacity.

Although oral iron supplementation may be economic and safe for treating RA-related anaemia, only $50 \%$ of the patients respond to this therapy (van Santen et al. 2014). Recent evidence has suggested that treatment of inflammation itself, may improve anaemia in RA. Indeed, anti-TNF therapy (Papadaki et al. 2002, Calisto Pérez et al. 2012, Sakthiswary et al. 2012, Furst et al. 2013) administered alone or combined with
DMARDs (Doyle et al. 2009) is suggested to improve haemoglobin levels in RA. However, little is known of LFN and ADA effects on anaemia. Indeed, a single study performed by Sakthiswary et al. (2012) recently demonstrated that RA patients treated with ADA exhibited improved levels of haemoglobin. Here, we present evidence on that both LFN and ADA treatments are able to attenuate anaemia in RA patients, denoted by increase in the levels of haemoglobin and decrease in the ESR. It is important to highlight that our study and the one by Sakthiswary et al. (2012) were performed in two very distinct populations (Malaysian $\mathrm{x}$ Brazilian), indicating that RA patients of different 
genetic backgrounds may benefit of ADA effects on anaemia. On the other hand, to the best of our knowledge, we present the first evidences on that LFN may be useful in treating RA-related anaemia.

Additionally, none of the treatments affected the number of red blood cells. Indeed, similar numbers of red blood cells were observed in all tested groups. These data suggest that anaemia in the evaluated patients is primarily related to increased iron retention or absorption within the reticulo-endothelial cells rather than haemolysis of erythropoietic progenitor cells.

We also found that the numbers of affected joints weakly correlate with the levels of haemoglobin in the NST group and in LFN- but not ADAtreated patients. Also, no significant correlations were observed between haemoglobin or ESR and the incidence of rheumatoid nodules. We suggest that anaemia may present a stronger correlation with other markers of disease activity, such as rheumatoid factor or C-reactive protein levels, pain, disability, amongst others. This hypothesis is supported by previous literature (Song et al. 2013).

LFN or ADA effects on anaemia may be related to their inhibitory actions on inflammation, including on cytokine generation. LFN is known to block $\mathrm{T}$ cell proliferation by inhibiting the synthesis of pyrimidine (Fragoso and Brooks 2015), and to reduce the activation of peripheral blood mononuclear cells (Grisar et al. 2004). ADA was also shown to reduce peripheral blood leukocyte (monocytes and PMNs) activation (Ríos-Navarro et al. 2015) and to inhibit T cell proliferation (Vos et al. 2011). All these suppressive effects on different cytokine-producing inflammatory cells may in turn be responsible for the attenuation of the anaemia in RA patients treated with LFN or ADA.

Overall, we present evidence on that treatment with LFN or ADA improves anaemia in RA, in addition to attenuating intra-articular damage, pain and disability. This is of importance as not all RA patients respond to iron supplementation.

\section{ACKNOWLEDGMENTS}

This work was supported by Coordenação de Aperfeiçoamento de Pessoal de Nível Superior (CAPES; grant 3325/2013), Conselho Nacional de Desenvolvimento Científico e Tecnológico (CNPq; 309046/2016-5), Fundação de Amparo à Pesquisa e ao Desenvolvimento Científico e Tecnológico do Maranhão (FAPEMA; grant 00311/14) and Farmácia Estadual de Medicamentos Especializados do Maranhão (FEME). V.L.P. Colares is an MSc student receiving a grant from FAPEMA. S.J.F. Mendes and D.M.S. Pereira are $\mathrm{PhD}$ students receiving grants from CAPES. The authors declare no conflict of interests.

\section{REFERENCES}

CALISTO PÉREZ C, LEÓN R, LEÓN F AND NG SL. 2012. Rheumatoid arthritis and anemia: the impact of different anti-inflammatory therapies on hemoglobin levels. An observational study. Bol Asoc Med P R 104: 34-41.

DOYLE MK, RAHMAN MU, HAN C, HAN J, GILES J, BINGHAM CO $3{ }^{\text {rd }}$ AND BATHON J. 2009. Treatment with infliximab plus methotrexate improves anemia in patients with rheumatoid arthritis independent of improvement in other clinical outcome measures-a pooled analysis from three large, multicenter, double-blind, randomized clinical trials. Semin Arthritis Rheum 39: 123-131.

FRAGOSO YD AND BROOKS JB. 2015. Leflunomide and teriflunomide: altering the metabolism of pyrimidines for the treatment of autoimmune diseases. Expert Rev Clin Pharmacol 8: 315-320.

FURST DE ET AL. 2013. The effect of golimumab on haemoglobin levels in patients with rheumatoid arthritis, psoriatic arthritis or ankylosing spondylitis. Rheumatology (Oxford) 52: 1845-1855.

GANNA S. 2014. The prevalence of anemia in rheumatoid arthritis. Rev Bras Reumatol 54: 257-259.

GRISAR J ET AL. 2004. Leflunomide inhibits transendothelial migration of peripheral blood mononuclear cells. Ann Rheum Dis 63: 1632-1637.

MASSON C. 2011. Rheumatoid anemia. Joint Bone Spine 78: 131-137.

MORELAND LW AND CURTIS JR. 2009. Systemic nonarticular manifestations of rheumatoid arthritis: focus on inflammatory mechanisms. Semin Arthritis Rheum 39: 132-143.

NYHÄLL-WÅHLIN BM ET AL. 2012. Extra-articular manifestations in a community-based sample of patients 
with rheumatoid arthritis: incidence and relationship to treatment with TNF inhibitors. Scand J Rheumatol 41: 434-437.

PAPADAKI HA, KRITIKOS HD, VALATAS V, BOUMPAS DT and ELIOPOULOS GD. 2002. Anemia of chronic disease in rheumatoid arthritis is associated with increased apoptosis of bone marrow erythroid cells: improvement following anti-tumor necrosis factor-alpha antibody therapy. Blood 100: 474-482.

RÍOS-NAVARRO C ET AL. 2015. Differential effects of anti-TNF- $\alpha$ and anti-IL-12/23 agents on human leukocyteendothelial cell interactions. Eur J Pharmacol 765: 355365.

SAKTHISWARY R, SYAHRUL SAZLIYANA S, MOHD SHAHRIR MS, SHAHRIL NS AND HUSSEIN H. 2012. Beyond the joints in rheumatoid arthritis: Effects of adalimumab on hematologic and lipid indices. EXCLI J 11:142-149.

SINGH H, ARYA S, TALAPATRA P, LATHER K, MATHUR R, SINGHANIA A AND CHAUDHARY V. 2014. Assessment of fatigue in rheumatoid arthritis (by Functional Assessment of Chronic Illness Therapy-Fatigue score) and its relation to disease activity and anemia. J Clin Rheumatol 20: 87-90.

SMYRNOVA G. 2014. The relationship between hemoglobin level and disease activity in patients with rheumatoid arthritis. Rev Bras Reumatol 54: 437-440.

SONG SN ET AL. 2013. Comparative evaluation of the effects of treatment with tocilizumab and TNF- $\alpha$ inhibitors on serum hepcidin, anemia response and disease activity in rheumatoid arthritis patients. Arthritis Res Ther 15: R141.

SPIVAK JL. 2000. The blood in systemic disorders. Lancet 355: 1707-1712.

VAN SANTEN S, DE MAST Q, OOSTING JD, VAN EDE A, SWINKELS DW AND VAN DER VEN AJ. 2014. Hematologic parameters predicting a response to oral iron therapy in chronic inflammation. Haematologica 99: e1713.

VOS AC, WILDENBERG ME, DUIJVESTEIN M, VERHAAR AP, VAN DEN BRINK GR AND HOMMES DW. 2011. Anti-tumor necrosis factor- $\alpha$ antibodies induce regulatory macrophages in an Fc region-dependent manner. Gastroenterology 140: 221-230. 